Astronomical Journal, in press

\title{
VLBA Identification of the Milliarcsecond Active Nucleus in the Seyfert Galaxy NGC 4151
}

\author{
James S. Ulvestad, ${ }^{1}$ Diane S. Wong, ${ }^{2}$ Gregory B. Taylor, ${ }^{3} \quad$ Jack F. Gallimore, ${ }^{4}$ and \\ Carole G. Mundell ${ }^{5}$
}

\begin{abstract}
The Seyfert galaxy NGC 4151 has been imaged at resolution better than 0.1 pc using a VLBI array consisting of the VLBA and three 100m-class telescopes. A flat-spectrum 3-mJy source with a monochromatic radio power of $\sim 10^{37}$ ergs s$^{-1}$ has been detected, apparently at the location of the active galactic nucleus (AGN) and its central black hole. The radio source has a minimum brightness temperature of $2.1 \times 10^{8} \mathrm{~K}$ and a size upper limit of $0.035 \mathrm{pc}$, about 10 times the diameter of the broad-line region and 15,000 times the diameter of the black hole's event horizon. An additional flat-spectrum component located within a parsec of the apparent nucleus is likely to be a knot in the inner radio jet. The presence of some steep-spectrum radio emission within $0.1 \mathrm{pc}$ of the galaxy nucleus limits the emission measure of a possible ionized torus to a maximum value of $10^{8} \mathrm{~cm}^{-6} \mathrm{pc}$. If the hard X-ray source in NGC 4151 is associated with the radio AGN, its radio to X-ray ratio is less than $10^{-5}$, putting NGC 4151 securely in the radio-quiet class of AGNs. The radio image reveals a 0.2 -pc two-sided base to the well-known arcsecond radio jet. Apparent speeds of jet components relative to the radio AGN are $<0.050 c$ and $<0.028 c$ at respective nuclear distances of $0.16 \mathrm{pc}$ and $6.8 \mathrm{pc}$. These are the lowest speed limits yet found for a Seyfert galaxy, and indicate non-relativistic jet motions, possibly due to thermal plasma, on a scale only an order of magnitude larger than the broad-line region.
\end{abstract}

\footnotetext{
${ }^{1}$ National Radio Astronomy Observatory, P.O. Box O, Socorro, NM 87801; julvesta@nrao.edu

${ }^{2}$ Department of Astronomy, University of California; dianew@astron.berkeley.edu

${ }^{3}$ National Radio Astronomy Observatory, P.O. Box O, Socorro, NM 87801; gtaylor@nrao.edu

${ }^{4}$ Department of Physics, Bucknell University, Lewisburg, PA 17837; jgallimo@bucknell.edu

${ }^{5}$ Astrophysics Research Institute, Liverpool John Moores University, Twelve Quays House, Egerton Wharf, Birkenhead, CH41 1LD, U.K.; cgm@astro.livjm.ac.uk
} 
Subject headings: galaxies: individual (NGC 4151) — galaxies: active — galaxies: Seyfert - radio continuum: galaxies

\section{Introduction}

A number of Seyfert galaxy radio sources have been imaged on milliarcsecond scales in recent years, using the Very Long Baseline Array (VLBA). Although each galaxy is unique, there are a number of common characteristics that have been revealed by VLBA imaging of Seyfert and LINER (Low Ionization Nuclear Emission-line Region) galaxies, as summarized by Ulvestad (2003): (1) Unresolved radio cores with flat or inverted spectra often are present (Wilson et al. 1998; Ulvestad, Wrobel, \& Carilli 1999a; Ulvestad et al. 1999b; Mundell et al. 2000; Falcke et al. 2000; Ulvestad \& Ho 2001; Nagar et al. 2002; Anderson, Ulvestad, \& Ho 2004); (2) Apparent relative motions in the few jets imaged at multiple epochs generally are quite subluminal (Roy et al. 1998; Ulvestad et al. 1998, 1999b; Roy et al. 2000), except during outbursts, when apparent speeds are seen to be as high as $0.5 c$ in Mrk 348 (Peck et al. 2003) and near $c$ in IIIZw2 (Brunthaler et al. 2000); and (3) At least some jets change their apparent directions of motion on scales of parsecs (Ulvestad et al. 1998, 1999b; Mundell et al. 2003). In the related radio-quiet quasars, there often are flat-spectrum cores with variability characteristics similar to the radio-loud quasars having relativistic jets (Barvainis et al. 2005). There have been no direct measurements of jet motions in radio-quiet quasars, though Blundell et al. (2003) have inferred superluminal motion in one object.

NGC 4151 was one of the original Seyfert galaxies classified in the 1940s (Seyfert 1943). Its optical spectra show both broad and narrow components in the Balmer emission lines, leading to a classification as a type 1.5 Seyfert galaxy (Osterbrock \& Koski 1976). The distance of NGC 4151, derived assuming a Hubble constant of $H_{0}=75 \mathrm{~km} \mathrm{~s}^{-1} \mathrm{Mpc}^{-1}$, is 20.3 Mpc (Tully 1988). ${ }^{6}$ This proximity and the consequent scale of $0.10 \mathrm{pc} \mathrm{mas}^{-1}$ make NGC 4151 one of the brightest and most well-studied Seyfert galaxies. Reverberation mapping of the broad emission lines shows that the radius of the broad-line region (BLR) is $\sim 2 \times 10^{-3} \mathrm{pc}$ (Wandel, Peterson, \& Malkan 1999), corresponding to an angular radius of only 0.02 mas. Assuming that the line widths are largely virialized around a central massive black hole, Wandel et al. (1999) determined a central black hole mass of $\simeq 1.2 \times 10^{7} M_{\odot}$.

\footnotetext{
${ }^{6}$ There are a range of distances cited in the literature for NGC 4151, typically varying from $\sim 13 \mathrm{Mpc}$ to $\sim 20 \mathrm{Mpc}$. We take a distance on the high end of this range because it has no impact on the spectral energy distribution (where all fluxes scale together), but does give the most conservative (high) values or limits to apparent component speeds.
} 
NGC 4151 contains a linear radio structure, or "jet," 3".5 (350 pc) in length at an average position angle of $77^{\circ}$. This structure has been imaged with progressively higher fidelity at sub-arcsecond resolution by the Very Large Array (VLA) (Wilson \& Ulvestad 1982; Johnston et al. 1982; Pedlar et al. 1993) and the Multi-Element Radio-Linked Interferometer Network (MERLIN) (Pedlar et al. 1993; Mundell et al. 1995), as well as being imaged with the European VLBI Network by Harrison et al. (1986). Five main radio components in the jet (C1 through $\mathrm{C} 5$ ) were identified in the MERLIN images, while milliarcsecond-resolution imaging using the VLBA resolved some of the MERLIN components and identified at least eight separate regions of radio emission, A-H (Ulvestad et al. 1998). The correspondence among the different radio components is shown in the top panel of Figure 1, reproduced from Mundell et al. (2003). The highest resolution 1.4 GHz VLBI images indicate that the average jet direction incorporates a number of sharp changes in position angle, possibly related to jet interactions with the circumnuclear environment (Mundell et al. 2003). In this paper, we discuss only the VLBA components $\mathrm{D}$ and $\mathrm{E}$, corresponding to $\mathrm{C} 4 \mathrm{~W}$ and $\mathrm{C} 4 \mathrm{E}$ in the MERLIN scheme; no other components were detected at our high resolution.

Mundell et al. (1995) hypothesized that the nucleus of the active galaxy is located in VLBA component D, based largely on the lack of $\mathrm{H}$ I absorption toward that radio component and the expectation that the inner part of any nuclear torus or accretion disk would be ionized by the active galactic nucleus (AGN). In contrast, Ulvestad et al. (1998) suggested that VLBA component E, located 7 pc further east, is the actual AGN, due to the possible presence of a subcomponent with a flat radio spectrum. However, high-resolution VLBA imaging of NGC 4151 recently has shown that all parts of component E exhibit $\mathrm{H}$ I absorption, making it unlikely that it includes the AGN, and therefore supporting the identification of component D as the galaxy nucleus (Mundell et al. 2003).

Hubble Space Telescope images of NGC 4151 with Wide Field Planetary Camera 1 (WFPC1) and with the Faint Object Camera revealed a $3^{\prime \prime}$ narrow line region (NLR) with a biconical form (Evans et al. 1993; Bokensberg et al. 1995); more details of the conical structure and the kinematics of the NLR have been revealed in the WFPC2 and Faint Object Spectrograph program carried out by Hutchings et al. (1998). Both a compact hard X-ray source and extended soft X-ray emission have been detected at or near the nucleus (Elvis, Briel, \& Henry 1983; Weaver et al. 1994; Yang, Wilson, \& Ferruit 2001). Near-infrared variability (Minezaki et al. 2004) and interferometric imaging (Swain et al. 2003) indicate the presence of a dust torus with inner radius of $\sim 0.05 \mathrm{pc}$, possibly with a geometrically thin accretion disk at smaller radius. Placing these small components in context, and studying the physical properties of the core of the AGN, is possible only if there is a secure identification of the radio nucleus of the galaxy. In this paper, we report high-sensitivity VLBI imaging of NGC 4151 that identifies the flat-spectrum radio nucleus of the galaxy and also provides the 
lowest upper limit to date on relative parsec-scale component speeds in any Seyfert galaxy.

\section{VLBI Observations and Imaging}

\subsection{Observations}

NGC 4151 was observed in dual-circular polarization at $5.0 \mathrm{GHz}$ and $8.4 \mathrm{GHz}(6 \mathrm{~cm}$ and $3.6 \mathrm{~cm}$ wavelengths, respectively), using the ten $25 \mathrm{~m}$ antennas of the VLBA (Napier et al. 1993) together with the phased VLA (twenty-seven 25m antennas), for a total of 12 hours on 1998 March 27 (program ID BG070). At each frequency, the total data rate employed was $128 \mathrm{Mbit} \mathrm{s}^{-1}$, yielding a total bandwidth of $16 \mathrm{MHz}$ at each polarization, with 2 bit sampling. In order to acquire more sensitivity in the search for a flat-spectrum core, NGC 4151 also was observed on 2002 May 16 (program BU022) for 10 hours, using a VLBI array consisting of the

VLBA, the phased VLA, the 100m Green Bank Telescope (GBT), and the 100m Effelsberg Telescope (Eb); the data rate and bandwidth were double those employed in 1998. In 2002, most of the observing time was spent at $15.3 \mathrm{GHz}$ (2 cm wavelength), with lesser amounts of time at 5.0 and $8.4 \mathrm{GHz}$. Due to the lengthy time required to reposition its subreflector in order to change frequency bands, the GBT observed only at $15.3 \mathrm{GHz}$.

Because NGC 4151 is too weak to detect fringes in an atmospheric coherence time, phasereferencing observations (Beasley \& Conway 1995) were employed, using the calibration source J1206+3941, approximately $2^{\circ}$ away from the galaxy. This calibrator now has an absolute position determined to 1 mas accuracy (Beasley et al. 2002) in the International Celestial Reference Frame (Ma et al. 1998). That position uncertainty dominates the absolute position errors for the NGC 4151 VLBI components, since ionospheric errors at $5 \mathrm{GHz}$ and higher should be less than 1 mas for a $2^{\circ}$ phase-reference distance (Chatterjee et al. 2004). Therefore, the absolute position error for NGC 4151 is taken to be 1 mas. The phasereferencing cycle time was about 12 minutes in 1998, and 2.5-4.5 minutes in 2002. A journal of the observations, including the total integration time on the galaxy, is provided in Table 1. Note that the need for frequent calibration meant that only about $35 \%$ of the scheduled 10 hours was spent actually integrating on the galaxy in 2002 .

\subsection{Calibration}

All data calibration was carried out in NRAO's Astronomical Image Processing System, AIPS (Greisen 2003). Amplitude calibration for the VLBA antennas in 1998 and 2002 was done by using the values for the antenna gains maintained by VLBA personnel as well as 
measurements of system temperature made once per minute during the observations; this calibration is believed to have an accuracy of 5\%. A similar method was used for calibration of Eb during 2002; approximately once per hour, that telescope was removed from the VLBI array in order to spend 15 minutes on pointing observations.

The 27 VLA antennas were made mutually coherent by adjusting their individual phases periodically during short observations of the compact sources J1206+3941 (in 1998) and J1146+3958 (in 2002). The amplitude response of the individual VLA antennas was calibrated with respect to the standard amplitude calibrator $\mathrm{J} 1331+3030$ (3C 286), on the scale of Baars et al. (1977), as modified slightly by the most recent measurements at the VLA. This amplitude calibration then was transferred to the reference source J1206+3941, enabling calculation of the complex gain, which was interpolated in time to derive the VLA calibration for NGC 4151.

For the GBT, we initially assumed a flat gain curve and $70 \%$ aperture efficiency based on commissioning measurements. This initial calibration was assessed using the amplitude check calibrators J0927+3902 and J1310+3220, and we found it necessary to reduce the amplitude (voltage) gain by $35 \%$ to bring the $15 \mathrm{GHz}$ calibration in line with the VLBA scale. Similar analysis resulted in a $10 \%$ gain reduction for $\mathrm{Eb}$, and small adjustments for several VLBA antennas, but no modifications to the a priori calibration were necessary at 5 and $8 \mathrm{GHz}$. For both 1998 and 2002, we estimate that the overall amplitude scale for NGC 4151 is uncertain $(1 \sigma)$ by $5 \%$ at $5 \mathrm{GHz}$ and $8.4 \mathrm{GHz}$. The significant amplitude corrections leave the fluxdensity scale at $15 \mathrm{GHz}$ somewhat more uncertain; based on "before" and "after" imaging of the amplitude-check source and the phase-reference source, we conservatively estimate the $1 \sigma$ uncertainty in the amplitude scale at $15 \mathrm{GHz}$ to be $10 \%$.

Initial clock offsets for all participating VLBI telescopes (including the phased VLA) were determined by means of phase-calibration tones and observations of the strong source J1642+3948 (3C 345). Time-dependent instrumental (and atmospheric) delays, delay rates, and phases were calibrated for J1206+3941 and interpolated to the observation times of NGC 4151. Due to the weakness of the galaxy and the fact that useful polarization calibration would have reduced the galaxy integration time further, no attempt was made to calibrate the instrumental polarization; VLA imaging (Ho \& Ulvestad 2001) indicates that the peak polarization signal on an arcsecond scale is $0.3 \mathrm{mJy}_{\text {beam }}^{-1}$ at $4.9 \mathrm{GHz}$. 


\subsection{Radio Imaging}

After the best possible initial calibration had been obtained, the calibrated data for NGC 4151 were imaged and self-calibrated using AIPS and Difmap (Shepherd 1997). Figure 1 shows the VLA-only image at $15.3 \mathrm{GHz}$, on arcsecond scales, from 2002, together with the VLBA 1.4-GHz image from Mundell et al. (2003). The VLA was in its BnA configura-

tion, yielding a resolution of $0^{\prime \prime} 32$ by $0^{\prime \prime} 15$. The VLA image shows the general structure of the arcsecond-scale jet; note that the entire VLBI structure discussed below, components D and $\mathrm{E}$, is found within the source labeled "Nucleus" in the VLA image. The 15.3-GHz flux density of this nucleus detected by the VLA is $28.1 \pm 1.4 \mathrm{mJy}$.

The VLBI imaging was carried out using a variety of weightings of the interferometric data; typically we used "natural" weighting, which optimizes the sensitivity, to produce the final images. This procedure is particularly important for the 2002 data, where such weighting gives the greatest strength to the data provided by the several large telescopes used along with the VLBA.

Figure 2 shows the final 5.0 GHz VLBI image from 1998.23, where significant emission was found at two locales separated by $\sim 70$ mas, or $7 \mathrm{pc}$. These components are those designated D3 and E by Ulvestad et al. (1998); components D1 and D2 are not detected. Respective noise values at 5.0 and $8.4 \mathrm{GHz}$ are 102 and $70 \mu \mathrm{Jy}_{\text {beam }}{ }^{-1}$ for the most sensitive images. Figure 3 displays blowups of components D3 and E at 5.0, 8.4, and $15.3 \mathrm{GHz}$, all from the data taken in 2002.37. Because of the inclusion of the large telescopes and additional bandwidth, the noise values are lower than those achieved in 1998, with respective values of 48,69 , and $45 \mu \mathrm{Jy}$ beam $^{-1}$ at $5.0,8.4$, and $15.3 \mathrm{GHz}$. Observation and imaging parameters are summarized in Table 1.

\section{Component Motions}

The nucleus of NGC 4151 is the origin of a two-sided radio jet that initially can be seen only within $\sim 0.2 \mathrm{pc}$ of the AGN (see upper right panel of Figure 3), but later is illuminated at various locations within $\sim 200 \mathrm{pc}$ of the nucleus (Mundell et al. 2003). The two-sided appearance of the jet and its side-to-side ratio near unity indicate that it is either nearly in the plane of the sky or is affected little by Doppler boosting. Since we apparently have a direct view of the broad line region in NGC 4151, we should be viewing the jet within $\sim 45^{\circ}$ of its axis, which would provide significant Doppler boosting and de-boosting of a jet moving near $c$. Therefore, indications just from the appearance of component D3 are that the local motions are considerably below $c$ within $0.2 \mathrm{pc}$ of the galaxy center. 
Ulvestad et al. (1998) found an upper limit of $0.14 c$ for the relative speed between the peaks of components D3 and E between 1984 and 1996. The new imaging in 1998 and 2002, at higher sensitivity than in 1996, enables a much more accurate measurement to be made. The component names in Table 2 represent an attempt to identify individual components that can be seen at both epochs, and whose apparent motions can be measured. However, it should be kept in mind that a generally similar appearance of images at the two epochs does not necessarily mean that the same physical components have been seen at both epochs.

The accuracy of the absolute positions of the various components in our VLBI images is limited by knowledge of the phase-reference source position and possible atmospheric effects over the $2^{\circ}$ distance between calibrator and reference source. However, the relative positions within NGC 4151 depend only to second order on these effects. The dominant quantifiable effect, instead, is simply the uncertainty in source position set by the limited signal/noise ratio (SNR) of the data. To zeroth order, the error in location of a component relative to some fiducial point in the image is equal to the full-width at half-maximum (FWHM) of the beam divided by the SNR of a component detection. Self-calibration may shift the absolute position of components in the image, but should not change the relative positions across the image, since all data for a given VLBI station have the same phase adjustments applied in the self-calibration process. Therefore, it is appropriate to use the SNR of the self-calibrated data in determining relative source position errors.

The above error analysis ignores possible systematic errors, such as source position shifts due to changing sensitivity to diffuse emission, frequency-dependent effects, and uncertainties in component identification. Apparently due to such effects, our fitting has shown relative position shifts of up to 0.05 mas (occasionally more) for different data weighting in the imaging, or between 8 and $15 \mathrm{GHz}$ fitting of peaks that appeared to be the same component. To account for this additional error source, we add 0.05 mas in quadrature to the SNRdetermined error and the fitting errors for each relative position measurement.

Here, note that we have been forced to add even more nomenclature, since component D3 splits into at least three components at $15 \mathrm{GHz}$, which we unimaginatively call D3a through D3c (from west to east). We measure position shifts relative to D3b, the most likely identification for the AGN (see Section 4). In 1998.42, the 8.4-GHz separation of D3b and E1 was $68.15 \pm 0.10$ mas, where we have used the beam size in the East-West direction (roughly the direction of separation) to compute the error. In 2002.37, the 15-GHz separation between the same two components was $68.33 \pm 0.06$ mas. Similarly, we can compute the separation on a much smaller scale, between D3b and D3c. This changed from $1.16 \pm 0.20$ mas to $1.56 \pm 0.06$ mas over the same 4-yr span; the large error in 1998.42 is caused by the difficulty in separating D3b and D3c at $8.4 \mathrm{GHz}$. 
On the face of it, the above analysis indicates possible detections of motion at slightly less than a $2 \sigma$ level in components located at $0.16 \mathrm{pc}$ and $6.8 \mathrm{pc}$ from the AGN. However, since the possible systematic and frequency-dependent effects are of somewhat uncertain magnitude, we would need at least a $3 \sigma$ result to be confident of a detection of motion in the radio components. Therefore, we quote $3 \sigma$ upper limits for the motion relative to D3b in $4.14 \mathrm{yr}$; these upper limits are 0.63 mas for component D3c and 0.35 mas for component E1, corresponding to respective velocity upper limits of $0.050 c$ and $0.028 c$. Assuming that the component identification across two epochs is correct, this is the lowest speed limit yet measured for any Seyfert galaxy. Of course, we must cite the usual caveat that we cannot distinguish motion (or lack thereof) of the actual radio-emitting material from motion (or lack thereof) of some structure such as a shock, which may stay near one position even while

material flows through it. Still, the very low limit of $\sim 15,000 \mathrm{~km} \mathrm{~s}^{-1}$ within $0.16 \mathrm{pc}$ of the AGN is not much larger than the gas velocities within the broad line region, and indicates that the jet in NGC 4151 may be dominated by thermal plasma in its innermost regions.

\section{Identification of the Active Nucleus}

At radio wavelengths, AGNs are most easily identified by the presence of a compact, flat- or inverted-spectrum core. Ulvestad et al. (1998) inferred that such a core was most likely to be present in component $\mathrm{E}$, in order to account for the slightly flattened radio spectrum attributed to component E. A principal aim of the present observations was to achieve considerably higher sensitivity to check for the presence of a weak core with a flat radio spectrum.

Inspection of Figure 3 immediately shows that component E contains no compact emission at $15 \mathrm{GHz}$ whose flux density is above $1 \mathrm{mJy}$. Since the peak flux densities at lower frequencies are much higher, there is no obvious flat-spectrum component that might be identified as the galaxy nucleus. However, component D3 contains an unresolved radio source with a flux density of approximately 3 mJy at $15 \mathrm{GHz}$, at the AGN location inferred by Mundell et al. (1995, 2003).

Of course, a visual impression is inadequate without a quantitative test for the presence of a flat-spectrum nucleus. The search for such a nucleus is complicated by the presence of several components in both D3 and E, combined with the quite different resolutions of the observations at frequencies varying by a factor of three. In addition, in component $\mathrm{E}$ at the low frequencies, the compact peaks clearly are embedded in smoother underlying emission that makes it challenging to isolate compact emission regions. 
We measured unresolved component flux densities by fitting Gaussian components to each peak in the images made with various data weightings and resolutions. Contamination from the underlying smooth emission was minimized by constraining the compact components to be unresolved. The fitted flux densities for images made with different data weighting are consistent with one another, so we use an average from the different images for each component. The combination of fitting and amplitude errors for individual components is estimated to be $7 \%$ at 5 and $8 \mathrm{GHz}$, and $12 \%$ at $15 \mathrm{GHz}$. These errors are larger than the amplitude scale uncertainty discussed in Section 2.2 because of increased confusion by the smooth emission underlying some compact components, as estimated by using component fits in images made with different data weighting. The increases of the component amplitude errors are fractionally larger at the lower frequencies, where the resolution is poorer and the underlying smooth flux is brighter. The final uncertainties in component flux densities were determined by taking the above errors from the amplitude scale and component fitting, then adding them in quadrature to the rms noise.

In order to test quantitatively for the presence of a flat-spectrum nucleus, we have tapered the weighting of the $15-\mathrm{GHz}$ data in the visibility plane in order to achieve resolution similar to that at $8 \mathrm{GHz}$. The resulting images (not shown, since they are similar to those in Figure 3) then were analyzed in two ways. First, we fit independent Gaussians to each significant component; results of those fits, including the relative source positions good to 0.01 mas, are presented in Table 2. In component $\mathrm{E}$, the radio emission is fairly smooth at 5 and $8 \mathrm{GHz}$, and shows a few local maxima at $15 \mathrm{GHz}$. Fitting individual compact components in component $\mathrm{E}$ was largely an exercise in intuition at the lower frequencies. Therefore, we have fitted the strongest emission component in E, calling it E1, but otherwise have not split component $\mathrm{E}$ into multiple subcomponents. E1 has a steep radio spectrum, with a two-point spectral index between 8 and $15 \mathrm{GHz}$ of $\alpha_{8,15}=-1.27 \pm 0.16$ (defining $\left.S_{\nu} \propto \nu^{+\alpha}\right)$. In contrast, D3b, the most powerful component of D3, has $\alpha_{8,15}=-0.11 \pm 0.23$. Component D3c has $\alpha_{8,15}=-0.48 \pm 0.30$, consistent with either a flat or a steep spectrum, while D3a is not clearly separable from D3b at $8 \mathrm{GHz}$, and therefore cannot be fitted.

The second method of analysis was to produce an image of the spectral index between 8 and $15 \mathrm{GHz}$. This was done by aligning the 8 and $15 \mathrm{GHz}$ images on the strongest peak of D3, then computing the spectral index at each individual pixel and generating a new spectralindex image; this image was blanked at all points where either of the input images had a flux density less than five times the noise level. The spectral indices then were evaluated along major axis slices through components D3 and E. Figure 4 shows the spectral index along the major axis slice of Component E. There clearly is no flat- or inverted-spectrum component here, so it is unlikely that Component E contains the nucleus of NGC 4151. 
Figure 5 shows the spectral index (top panel) and the $15 \mathrm{GHz}$ total intensity (bottom panel) along the major axis (in the direction from West to East) of Component D3; the locations of the three sub-components in D3 are indicated in the figure. It appears that there is flat-spectrum emission near all three radio components. We note, however that the major axis slice does not intersect the peak of D3c, which is considerably north of the major axis defined by the two stronger components. The apparent flat spectrum near D3c appears to be an artifact of the fact that the slice actually intersects the edge of the radio component, where the SNR is quite low and the spectrum probably is affected by component registration errors that are a small fraction of a beam in size.

We then must assess the spectra of D3a and D3b from the spectral slices. The spectralindex image was produced by aligning the $8 \mathrm{GHz}$ and $15 \mathrm{GHz}$ peaks, in D3b, to an accuracy of about 1 microarcsecond. Assuming that this alignment is correct, the spectral index at the exact location of D3b can be taken from the spectral-index slice, and is found to be $\alpha_{8,15}=+0.16 \pm 0.23$, consistent with the value of $-0.11 \pm 0.23$ that was found from the gaussian fitting. At D3a, we find a similar spectral index of $\alpha_{8,15}=+0.20 \pm 0.24$. This cannot be compared with the value from a gaussian fit because the 8-GHz image has no distinct peak at the location of D3a. We note that taking single values from a spectral index slice is a perilous endeavor, because this process amounts to assuming both infinite resolution and perfect alignment of the two input images. The beam size along the major axis of D3 is 0.5 mas, as plotted in Figure 5, and the absolute alignment of the $8 \mathrm{GHz}$ and $15 \mathrm{GHz}$ images probably is known to no better than 0.1-0.2 mas. Therefore, the best conclusions that can be drawn from the spectral-index slices are that D3a and D3b represent the location of two flat-spectrum components separated by less than twice the image resolution, and that there appears to be weak steep-spectrum emission between them.

A similar analysis to that described above might be carried out using the data at $5 \mathrm{GHz}$ as well. However, there is insufficient resolution of D3 to distinguish the separate components, which causes any possible nuclear source to be blended with surrounding emission. If we simply use the values in Table 2 , we find $\alpha_{5,15}=-0.26 \pm 0.13$ for component D3b. Since the $5-\mathrm{GHz}$ flux density includes some confusing flux from component D3c, the true spectral index of D3b is probably less steep (more positive, or less negative) than the computed value.

The spectral data strongly support the inference of Mundell et al. (1995, 2003) that component D3 contains the true AGN in NGC 4151. D3b is the strongest flat-spectrum component, by about a factor of four. In addition, the two-sidedness of the arcsecond-scale jet (see Figure 1), as well as the predominance of two-sided jets in low-power radio sources (Giovannini et al. 2005) make it likely that the smallest scale jet should be two-sided rather than one-sided. These factors imply that D3b is the most likely AGN identification, and 
such an identification will be assumed below. However, the reader should bear in mind that it is conceivable that the AGN might be located at D3a. In that case, the nuclear radiosource size for self-absorption (see below) would be smaller by about a factor of two, the innermost jet would be one-sided, and the nuclear radio/X-ray ratio would be smaller by a factor of about four. Mundell et al. (2003) suggested that NGC 4151 might be similar to the compact symmetric objects seen in more distant radio galaxies, but the apparent lack of any flat-spectrum component between D3a and D3b makes it highly unlikely that they represent jet components on opposite sides of an undetected radio nucleus.

Absolute J2000 coordinates of D3b, are $\alpha=12 \mathrm{~h} 10 \mathrm{~m} 32.5758 \mathrm{~s}, \delta=39^{\circ} 24^{\prime} 21.060^{\prime \prime}$, accurate to 1 mas. This radio nucleus should be at the apex of the "cone" of narrow-line clouds imaged with HST (Evans et al. 1993; Bokensberg et al. 1995; Hutchings et al. 1998), and presumably at the center of the broad-line region (Wandel et al. 1999) as well as the center of the accretion disk and dust torus detected in the near infrared (Swain et al. 2003; Minezaki et al. 2004). Since the Gaussian fit of D3b indicates that it is unresolved, we take an upper limit of half the highest-resolution beam size in each dimension, yielding a brightness temperature of $T_{\mathrm{B}}>2.1 \times 10^{8} \mathrm{~K}$. This is consistent with, but does not require, the possibility that synchrotron self-absorption could produce the flat radio spectrum. The angular upper limit of 0.35 mas (averaging the major- and minor-axis limits) for the radio source size corresponds to a linear diameter of less than 0.035 pc. This is only $\sim 10$ times the diameter of the BLR, but about 15,000 times the diameter of the event horizon of the supermassive black hole. If we assume that the flat spectrum is due to synchrotron self-absorption, and that this requires $T_{\mathrm{B}} \gtrsim 10^{10} \mathrm{~K}$, the upper limit to the radio source size would be very close to the diameter of the BLR, or $\sim 4 \times 10^{-3}$ pc. This limit of about 2000 gravitational radii is comparable to the limits for several low-luminosity AGNs in galaxies found at similar distances (Ulvestad \& Ho 2001; Anderson et al. 2004).

The apparent flat-spectrum component D3a, which appears relatively prominent at 15 $\mathrm{GHz}$ and not obvious at $8.4 \mathrm{GHz}$, has a lower brightness temperature limit of $T_{\mathrm{B}}>5 \times 10^{7} \mathrm{~K}$. This component could well represent a shock in the inner jet, less than $0.1 \mathrm{pc}$ from the AGN. One might speculate that D3a could be a location where the jet collides with material in the inner narrow-line region, as may be seen on larger scales (Mundell et al. 2003). However, the inability of optical emission-line imaging to probe a scale similar to that seen by VLBI makes it difficult to test this possibility.

An alternative hypothesis for the flat-spectrum radio components would be that their spectral shapes are caused by free-free absorption by ionized gas in the inner torus, lying in front of synchrotron-emitting plasma. Since D3a and D3b have fairly flat spectra between 8 and $15 \mathrm{GHz}$, the presence of free-free absorption would imply an optical depth $\tau_{\mathrm{ff}} \approx 1$ 
at a frequency of $\sim 10 \mathrm{GHz}$; for a gas temperature near $8000 \mathrm{~K}$, this implies an emission measure of $n_{e}^{2} d L \approx 3 \times 10^{8} \mathrm{~cm}^{-6} \mathrm{pc}$. If all of component D3 were enveloped in an ionized torus of radius $\sim 3 \mathrm{pc}$ (Mundell et al. 2003), then the average density in that torus (for a filling factor of unity) would be $\sim 10^{4} \mathrm{~cm}^{-3}$, somewhat below the value inferred by Mundell et al. (2003). However, it seems apparent that there is some steep-spectrum emission in D3 (e.g., between D3a and D3b, as shown in Figure 5). This would imply a very patchy torus, and would make one wonder why the densest ionized gas "coincidentally" lies in front of the radio components. Thus, the more likely scenario for free-free absorption, if it caused the flat spectra, might be an ionized "skin" around the radio components. This thermal gas would have a path length shorter than $0.1 \mathrm{pc}$, and therefore an ionized density $n_{e} \gtrsim 10^{5} \mathrm{~cm}^{-3}$. In fact, we consider the most likely inference to be that we have detected synchrotron selfabsorption and no free-free absorption. Then, the presence of some steep-spectrum radio emission at $8 \mathrm{GHz}$ simply limits the overall emission measure of a quasi-uniform ionized medium to $\lesssim 10^{8} \mathrm{~cm}^{-6} \mathrm{pc}$.

We note that the flux density measured for component D3 at $15.3 \mathrm{GHz}$ is above the upper limit previously reported by Ulvestad et al. (1998). The difference might result from source variability by a factor of two, certainly a viable possibility given the strong variability of the hard and medium-energy X-rays from the galaxy nucleus (Perotti et al. 1981; Weaver et al. 1994). However, the 1996.43 VLBI phase-referencing observations (Ulvestad et al. 1998) also could have suffered from substantial loss of atmospheric coherence. In 2002.37, there was significant coherence loss in the initial phase referencing, which was recovered by the use of the large telescopes both for the initial detection of component D3 and for the subsequent self-calibration. Therefore, the present data seem to be consistent with the previous upper limit of $2.2 \mathrm{mJy}_{\text {beam }}^{-1}$ at $15 \mathrm{GHz}$, once coherence losses are taken into account.

The total flux density in VLBI-detected components is only $6.4 \pm 0.8 \mathrm{mJy}$ at $15 \mathrm{GHz}$; this number was derived by adding the individual fits in component $\mathrm{D}$ to the integrated flux density for the entire emission region in E. This is only $\sim 20 \%-25 \%$ of the total core flux density seen by the VLA (see Figure 1), with only $10 \%$ of the total VLA flux density found in the putative AGN. Therefore, much of the unresolved VLA 15-GHz emission must be in jet components that are resolved out by the VLBA. This result points out the peril of using even unresolved VLA flux densities in modeling the emission processes from AGNs, since the spectral energy distribution of the actual milliarcsecond AGN, and the radio/X-ray ratio, would be in error by an order of magnitude if the unresolved VLA flux were used.

The 2-9 keV X-ray flux detected by the Chandra X-ray satellite is $4.8 \times 10^{-11} \mathrm{ergs} \mathrm{cm}^{-2} \mathrm{~s}^{-1}$ (Yang et al. 2001), corresponding to a luminosity of $2.3 \times 10^{42} \mathrm{ergs} \mathrm{s}^{-1}$. Using a factor of 
$\sim 7$ to convert from X-ray to bolometric luminosity (Ho 1999; Ho et al. 2000), this implies that the nucleus of NGC 4151 is radiating at about $1 \%$ of its Eddington luminosity of $1.6 \times 10^{45} \mathrm{ergs} \mathrm{s}^{-1}$ for the $1.2 \times 10^{7} M_{\odot}$ black hole inferred by Wandel et al. (1999). Component D3b has a flux density of $\sim 4 \mathrm{mJy}$ at $5 \mathrm{GHz}$, and a fairly flat spectrum, implying a monochromatic 5-GHz luminosity of $\nu S_{\nu} \sim 1 \times 10^{37} \mathrm{ergs} \mathrm{s}^{-1}$. Given that the quoted X-ray luminosity was near the minimum for a source that varies by about a factor of 10 (Weaver et al. 1994; Yang et al. 2001), the ratio of radio to hard X-ray luminosities is given by $R_{X}=\log \left[\nu S(5 \mathrm{GHz}) / F_{X}(2-10 \mathrm{keV})\right] \approx-5.5$ to -6.5 , roughly two orders of magnitude lower than the similar ratio for several low-luminosity active galaxies (Ulvestad \& Ho 2001; Terashima \& Wilson 2003). $R_{X}$ is clearly much less than -4.7 , the dividing line below which an AGN such as NGC 4151 would be considered radio-quiet (Terashima \& Wilson 2003). ${ }^{7}$

\section{Conclusions}

We have imaged the core of the Seyfert galaxy NGC 4151 at two epochs and multiple frequencies with a VLBI array consisting of the VLBA and several 100m-class radio telescopes. The images reveal a compact flat-spectrum radio component having $T_{\mathrm{B}}>2.1 \times 10^{8} \mathrm{~K}$, which we identify as the actual location of the active galactic nucleus and its 12-million solar mass black hole; this component is at the location inferred by Mundell et al. (1995, 2003). The radio flux density of $\sim 3-4$ mJy corresponds to a monochromatic power of $\sim 10^{37} \mathrm{ergs} \mathrm{s}^{-1}$ at 5-15 GHz; comparison to the X-ray luminosity indicates that NGC 4151 is a radio-quiet object, in contrast to a number of other low luminosity active galaxies. A weak, two-sided beginning to the larger scale radio jet is seen to exist well within the inner parsec of the AGN. Upper limits to the component speeds relative to the apparent core are $0.050 c$ and $0.028 c$ at respective distances of $0.16 \mathrm{pc}$ and $6.8 \mathrm{pc}$ from the AGN, implying that the NGC 4151 jet is non-relativistic, and dominated by thermal plasma, all the way down to near the broad-line region. This is consistent with the approximately symmetric radio morphology about the apparent center of activity.

The National Radio Astronomy Observatory is a facility of the National Science Foundation operated under cooperative agreement by Associated Universities, Inc. We thank the staffs of the VLA, VLBA, GBT, and Effelsberg telescopes that made these observations

\footnotetext{
${ }^{7}$ The reader should keep in mind that the X-ray and radio strengths were not measured at the same epoch, and that there is no conclusive observational evidence for or against the possibility that the weak radio core might vary by factors of $2-3$. However, an increase by a factor of three in the radio core still would leave it in the radio-quiet regime as defined by the radio/X-ray ratio.
} 
possible; we are especially grateful to Frank Ghigo for his efforts in making these VLBI observations a success very soon after the $15 \mathrm{GHz}$ capability first became available at the GBT. DSW acknowledges support from the NRAO summer research program and from the National Science and Engineering Research Council of Canada. CGM acknowledges financial support from the Royal Society. We thank the anonymous referee for very useful suggestions about the analysis of the nuclear radio spectrum and other issues.

\section{REFERENCES}

Anderson, J. M., Ulvestad, J. S., \& Ho, L. C. 2004, ApJ, 603, 42

Baars, J. W. M., Genzel, R., Pauliny-Toth, I. I. K., \& Witzel, A. 1977, A\&A, 61, 99

Barvainis, R., Lehár, J., Birkinshaw, M. Falcke, H. \& Blundell, K.M. 2005, ApJ, 618, 108

Beasley, A. J., \& Conway, J. E. 1995 in ASP Conf. Ser. 1982, Very Long Baseline Interferometry and the VLBA, Ed. J. A. Zensus, P. J. Diamond, \& P. J. Napier (San Francisco: ASP), 327

Beasley, A. J., Gordon, D., Peck, A. B., Petrov, L., MacMillan, D. S., Fomalont, E. B., \& Ma, C. 2002, ApJS, 141, 13

Blundell, K. M., Beasley, A. J., \& Bicknell, G. V. 2003, ApJ, 591, L103

Bokensberg, A., et al. 1995, ApJ, 440, 151

Brunthaler, A., et al. 2000, A\&A, 357, L45

Chatterjee, S., Cordes, J. M., Vlemmings, W. H. T., Arzoumanian, Z., Goss, W. M., \& Lazio, T. J. W. 2004, ApJ, 604, 339

Elvis, M., Briel, U. G., \& Henry, J. P. 1983, ApJ, 268, 105

Evans, I. N., Tsvetanov, Z., Kriss, G. A., Ford, H., C., Caganoff, S., \& Koratkar, A. P. 1993, ApJ, 417, 82

Falcke, H., Nagar, N. M., Wilson, A. S., \& Ulvestad, J. S. 2000, ApJ, 542, 197

Giovannini, G., Taylor, G. B., Feretti, L., Cotton, W. D., Lara, L., \& Venturi, T. 2005, ApJ, 618,635

Greisen, E. W. 2003, in Information Handling in Astronomy - Historical Vistas, ed. A. Heck, Astrophysics and Space Science Library Vol. 285 (Dordrecht: Kluwer), 109 
Harrison, B., Pedlar, A., Unger, S. W., Burgess, P., Graham, D. A., \& Preuss, E. 1986, MNRAS, 218, 775

Ho, L. C. 1999, ApJ, 516, 672

Ho, L. C., Rudnick, G., Rix, H.-W., Shields, J. C., McIntosh, D. H., Filippenko, A. V., Sargent, W. L. W., \& Eracleous, M. 2000, ApJ, 541, 120

Ho, L. C., \& Ulvestad, J. S. 2001, ApJS, 133, 77

Hutchings, J. B., et al. 1998, ApJ, 492, L115

Johnston, K. J., Elvis, M., Kjer, D., \& Shen, B. S. P. 1982, ApJ, 262, 61

Ma, C., et al. 1998, AJ, 116, 516

Minezaki, T., Yoshii, Y., Kobayashi, Y., Enya, K., Suganuma, M., Tomita, H., Aoki, T., \& Peterson, B. A. 2004, ApJ, 600, L35

Mundell, C. G., Pedlar, A., Baum, S. A., O’Dea, C. P., Gallimore, J. F., \& Brinks, E. 1995, MNRAS, 272, 355

Mundell, C. G., Wilson, A. S., Ulvestad, J. S., \& Roy, A. L. 2000, ApJ, 529, 816

Mundell, C. G., Wrobel, J. M., Pedlar, A., \& Gallimore, J. F. 2003, ApJ, 583, 192

Nagar, N. M., Falcke, H., Wilson, A. S., \& Ulvestad, J. S. 2002, A\&A, 392, 53

Napier, P. J., Bagri, D. S., Clark, B. G., Rogers, A. E. E., Romney, J. D., Thompson, A. R., \& Walker, R. C. 1993, Proc IEEE, 82, 658

Osterbrock, D.E., \& Koski, A.T. 1976, MNRAS, 176, 61P

Peck, A. B., Henkel, C., Ulvestad, J. S., Brunthaler, A., Falcke, H., Elitzur, M., Menten, K. M., \& Gallimore, J. F. 2003, ApJ, 590, 149

Pedlar, A., Kukula, M. J., Longley, D. P. T., Muxlow, T. W. B., Axon, D. J., Baum, S., O’Dea, C., \& Unger, S. W. 1993, MNRAS, 263, 471

Perotti, F., della Ventura, A., Villa, G., di Cocco, G., Bassani, L., Butler, R. C., Carter, J. N., \& Dean, A. J. 1981, ApJ, 247, L63

Roy, A. L., Colbert, E. J. M., Wilson, A. S., \& Ulvestad, J. S. 1998, ApJ, 504, 147 
Roy, A. L., Wilson, A. S., Ulvestad, J. S., \& Colbert, E. J. M. 2002, in Proceedings of the 5th EVN Symposium, Ed. J. E. Conway, A. G. Polatidis, R. S. Booth, \& Y. M. Pihlström (Onsala: Onsala Space Observatory), 7

Seyfert, C. K. 1943, ApJ, 97, 28

Shepherd, M. C. 1997, in Astronomical Data Analysis Software and Systems VI, ASP Conf. Ser. 125, Ed. G. Hunt \& H. E. Payne (San Francisco: ASP), 77

Swain, M., et al. 2003, ApJ, 596, L163

Terashima, Y., \& Wilson, A. S. 2003, ApJ, 583, 145

Tully, R. B. 1988, Nearby Galaxies Catalog (Cambridge: Cambridge University Press)

Ulvestad, J. S. 2003, in Radio Astronomy at the Fringe, ASP Conf. Ser. 300, Ed. J. A. Zensus, M. H. Cohen, \& E. Ros (San Francisco: ASP), 97

Ulvestad, J. S., \& Ho, L. C. 2001, ApJ, 562, L133

Ulvestad, J. S., Roy, A. L., Colbert, E. J. M., \& Wilson, A. S. 1998, ApJ, 496, 196

Ulvestad, J. S., Wrobel, J. M., \& Carilli, C. L. 1999a, ApJ, 516, 127

Ulvestad, J. S., Wrobel, J. M., Roy, A. L., Wilson, A. S., Falcke, H., \& Krichbaum, T. P. 1999b, ApJ, 517, L81

Wandel, A., Peterson, B. M. \& Malkan, M. A. 1999, ApJ, 526, 579

Weaver, K. A., Yaqoob, T., Holt, S. S., Mushotzky, R. F., Matsuoka, M., \& Yamauchi, M. 1994, ApJ, 436, L27

Wilson, A. S., \& Ulvestad, J. S. 1982, ApJ, 263, 576

Wilson, A. S., et al. 1998, ApJ, 505, 587

Yang, Y., Wilson, A. S., \& Ferruit, P. 2001, ApJ, 563, 124

This preprint was prepared with the AAS LATEX macros v5.2. 

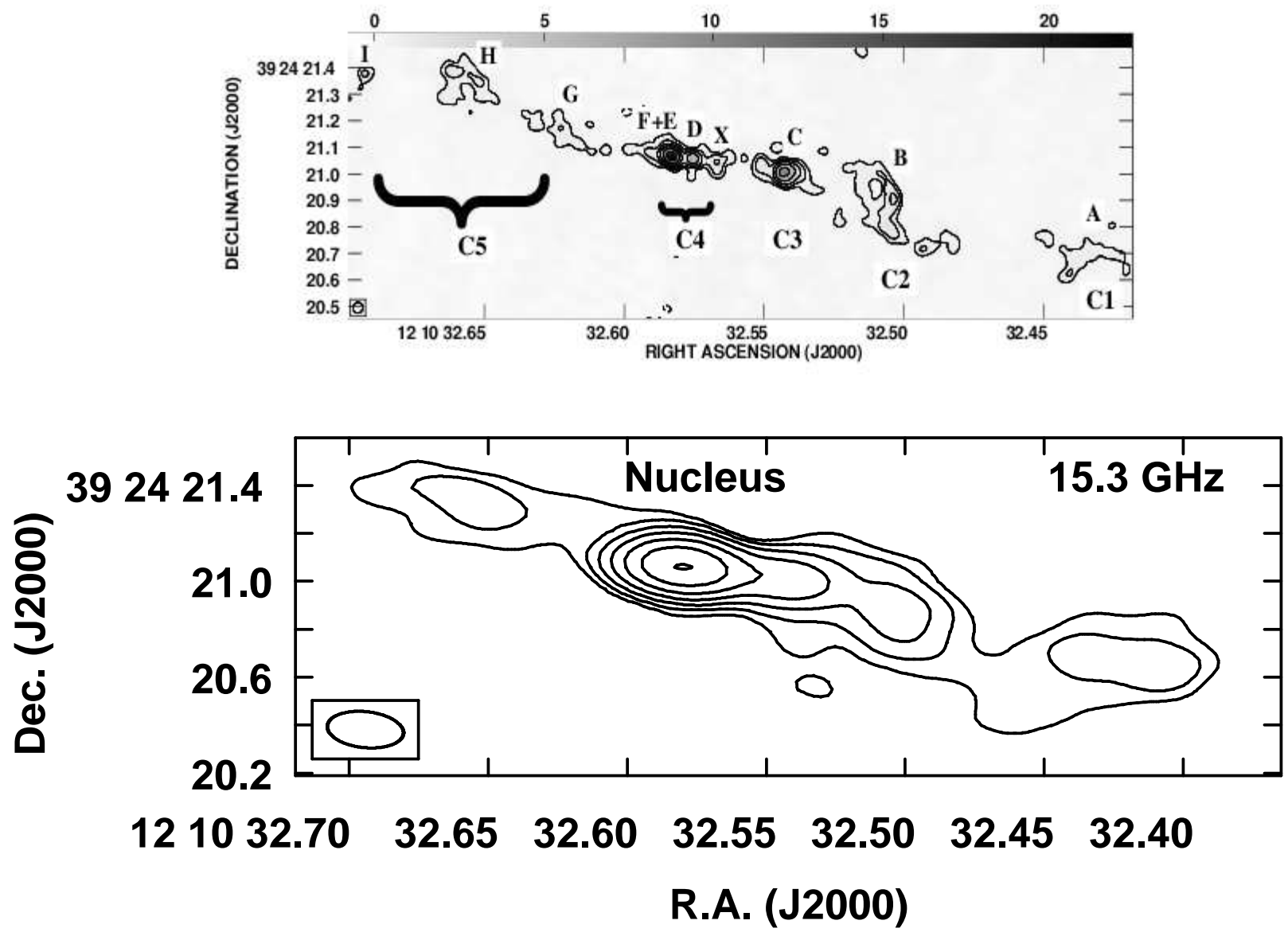

Fig. 1.- Top: VLBA Image of NGC 4151 jet, from Mundell et al. (2003), showing the nomenclature for the radio components. Bottom: $15 \mathrm{GHz}$ VLA image of NGC 4151 at epoch 2002.37 from the current observations. Components, X, D, E, and F from the upper panel all are included in the component marked "Nucleus" in the bottom panel. The restoring beam for the VLA image is $0^{\prime \prime} 32$ by $0^{\prime \prime} 15$ in position angle $84^{\circ}$. Contour levels begin at 3 times the rms noise of $141 \mu \mathrm{Jy}_{\text {beam }}{ }^{-1}$ and increase by factors of two. The scale of the VLA image differs slightly from the VLBA image, due to the much larger beam size. 


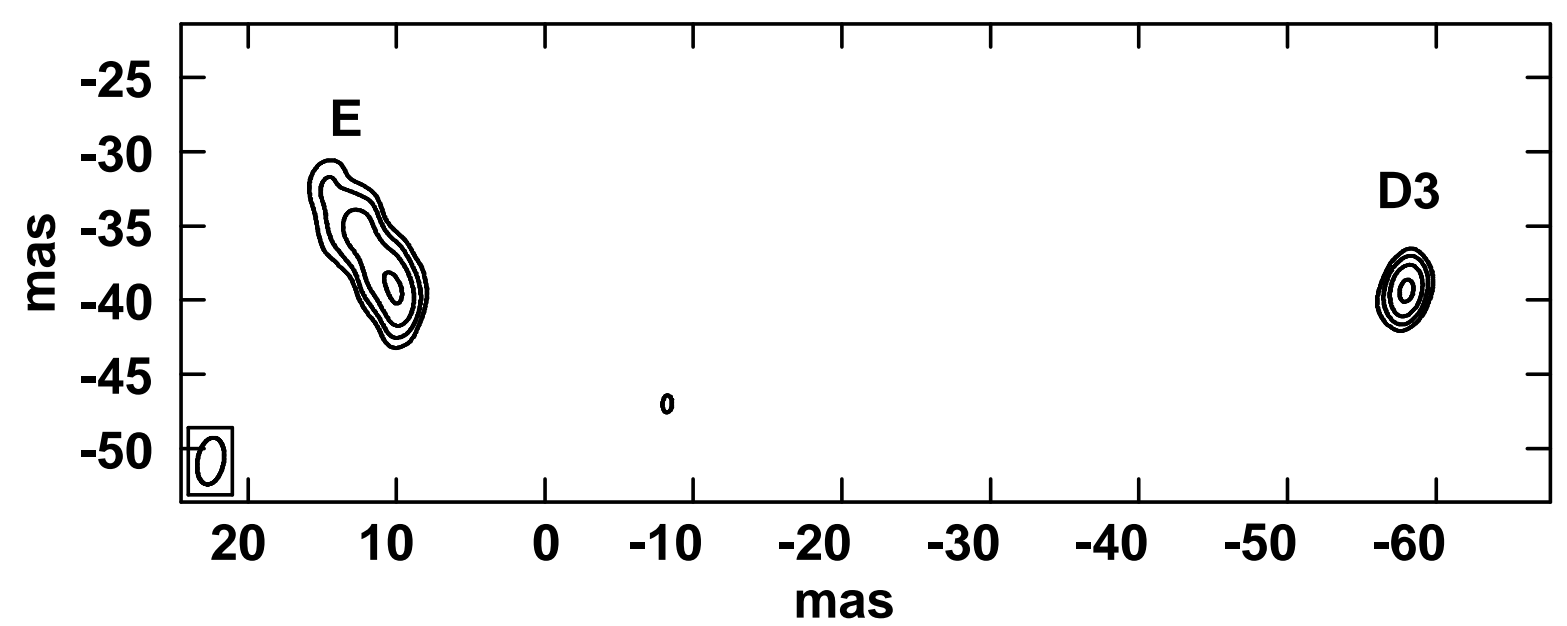

Fig. 2.- VLBI image of NGC 4151 at $5.0 \mathrm{GHz}$, from epoch 1998.24. The restoring beam is $3.2 \times 1.8$ mas in position angle $-10^{\circ}$. Contour levels begin at 3 times the rms noise of $102 \mu \mathrm{Jy}^{\text {beam }}{ }^{-1}$ and increase by factors of two. Coordinates are given relative to $\alpha=12 \mathrm{~h} 10 \mathrm{~m} 32.5831 \mathrm{~s}, \delta=39^{\circ} 24^{\prime} 21.099^{\prime \prime}$ (the original pointing position plus an offset due to the updated position of the reference source J1206+3941). 

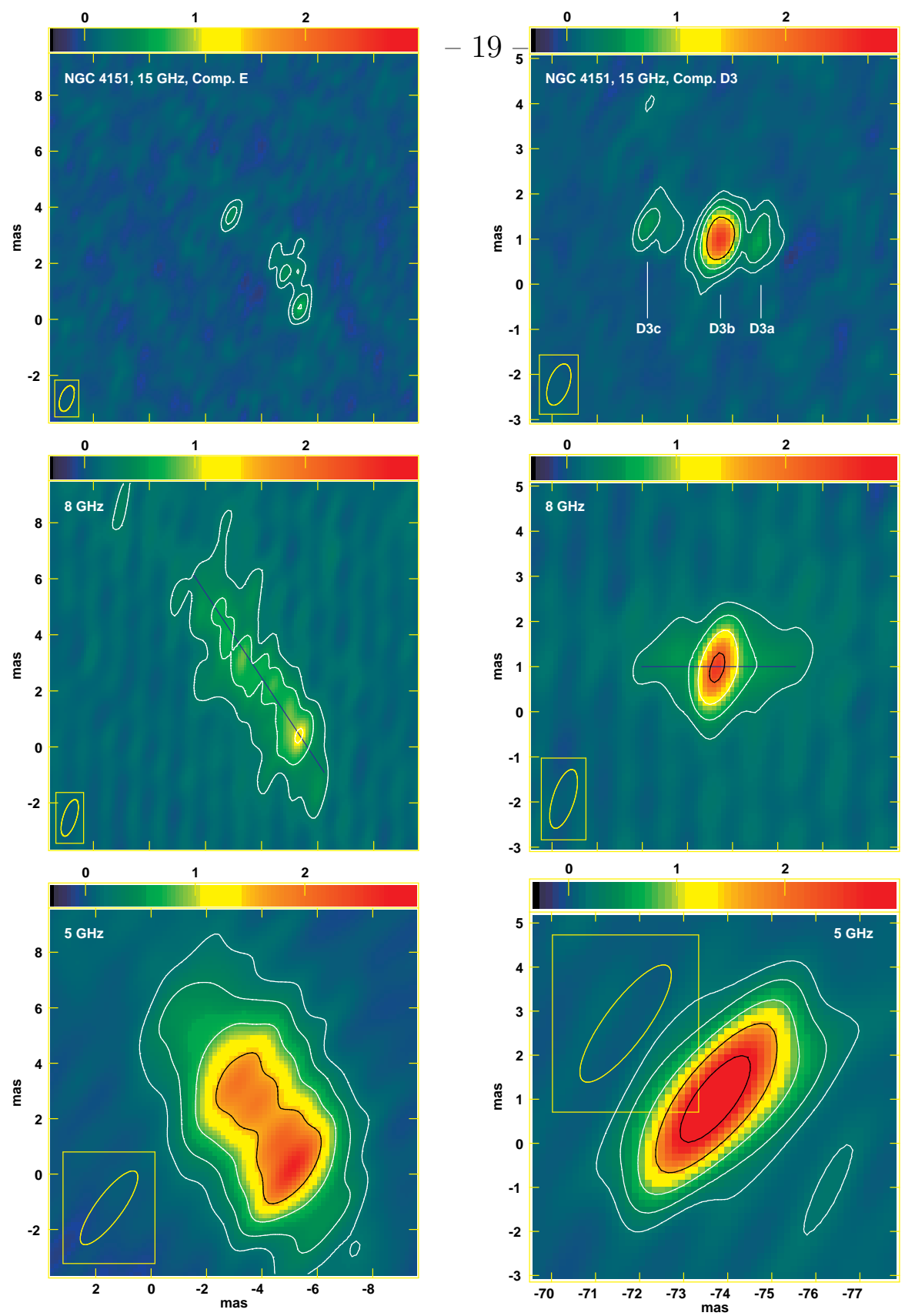

Fig. 3.- From top to bottom, 15.3, 8.4, and 5.0 GHz VLBI images of NGC 4151 are shown at epoch 2002.37. Contour levels begin at 4 times the rms noises given in Table 1 and increase by factors of two. Beam sizes are given in Table 1 and shown by the boxed ellipses in lower or upper left-hand corners. Component $\mathrm{E}$ is shown in the left panels, with D3 in the right panels, at a somewhat different scale. The lines drawn along the major axes of the 8.4 GHz images indicate the positions of the slices shown in Figures 4 and 5. All coordinates are given in milliarcseconds offset from the original pointing position of $\alpha=12 \mathrm{~h} 10 \mathrm{~m} 32.5822 \mathrm{~s}$, $\delta=39^{\circ} 24^{\prime} 21.059^{\prime \prime}$. All images have the same flux-density color scale, shown by the bar at the top of each image. 


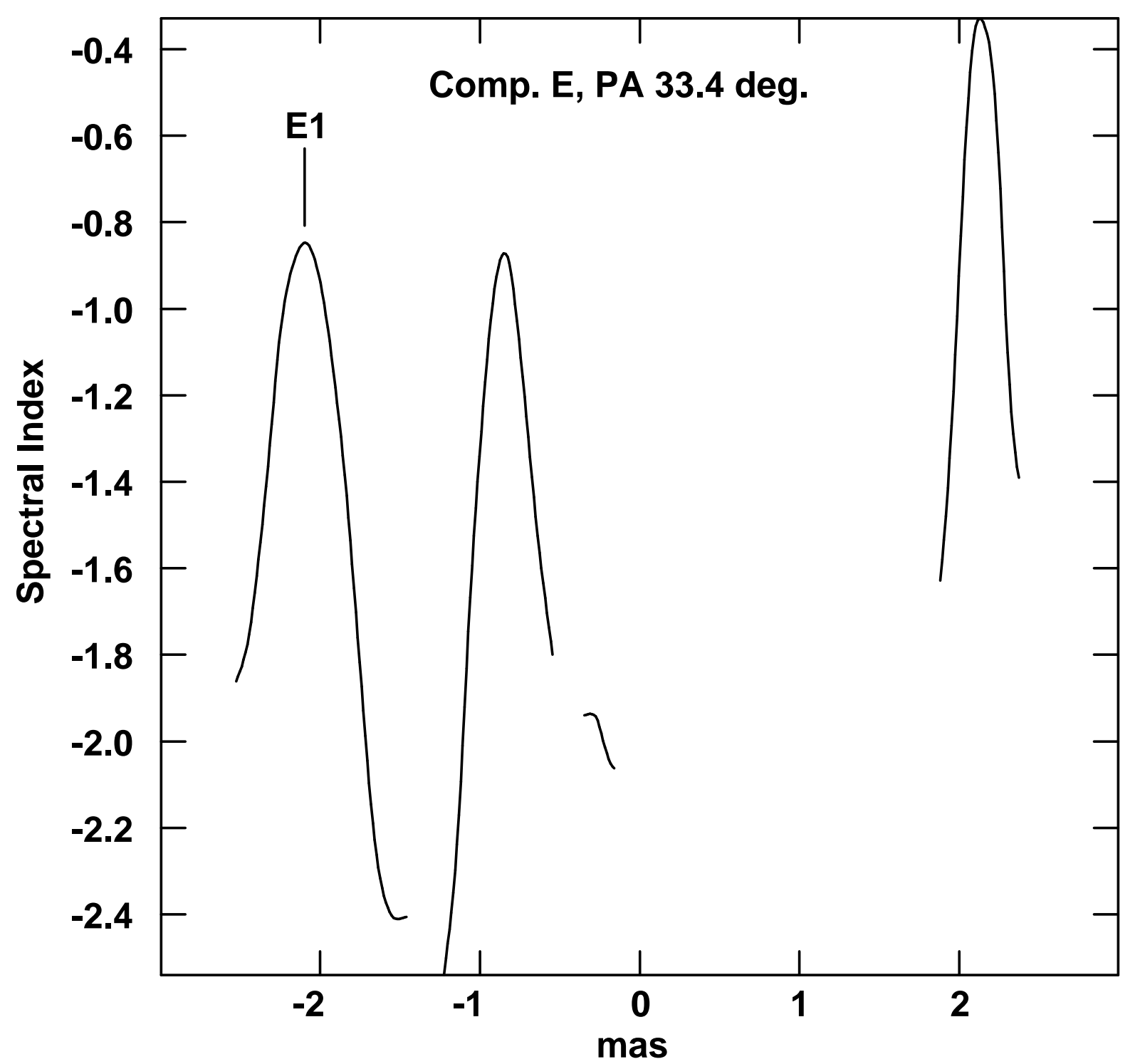

Fig. 4.- Two-point spectral index along the major axis of component $\mathrm{E}$ between 8.4 and 15.3 $\mathrm{GHz}$ at 2002.37, using the $15.3 \mathrm{GHz}$ image smoothed to the 8.4-GHz resolution. Distances are labeled in milliarcseconds relative to the center of the slice, and the position of E1 is indicated. The spectral index map has been blanked where the flux density is below the $5 \sigma$ detection threshold in either input image. The slice goes from SW to NE, in position angle $33.4^{\circ}$. 


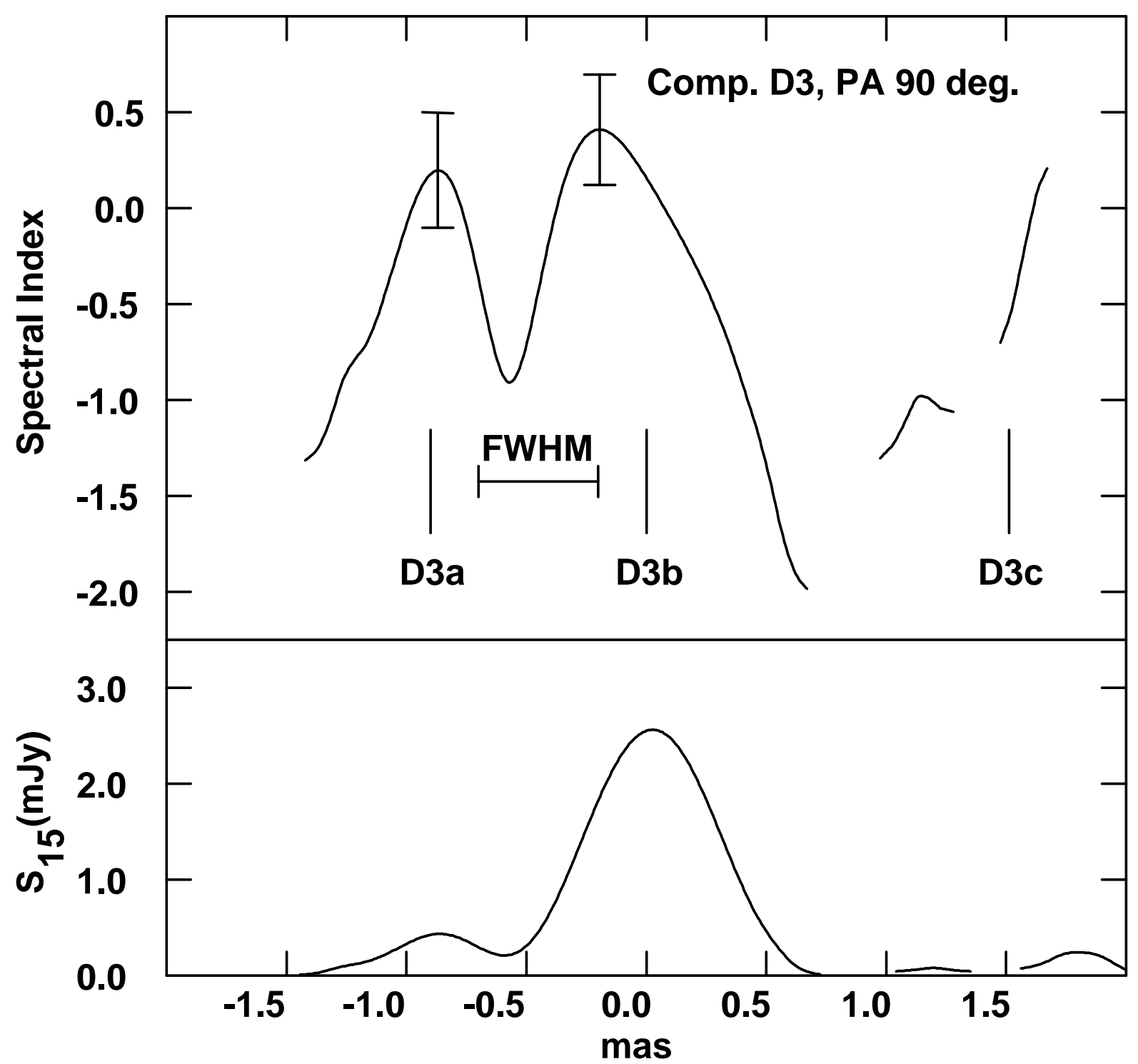

Fig. 5.- Slices of two-point spectral index (top panel) and 15-GHz flux density (bottom panel) along the major axis of component D3, in position angle $90.0^{\circ}$, using the same images as in Figure 4. Positions of the three sub-components of D3 are shown, as are the representative $(1 \sigma)$ spectral-index errors and the full width at half maximum (FWHM) of the image resolution along the slice. Note that the entire slice is only a few resolution elements in length so small features should be interpreted with extreme caution. 
Table 1. VLBI Observations of NGC 4151

\begin{tabular}{|c|c|c|c|c|c|}
\hline $\begin{array}{c}(1) \\
\text { Date }\end{array}$ & $\begin{array}{c}(2) \\
\text { Frequency } \\
(\mathrm{GHz})\end{array}$ & $\begin{array}{c}(3) \\
\text { Telescopes }\end{array}$ & $\begin{array}{l}(4) \\
\text { Integration } \\
(\min )\end{array}$ & $\begin{array}{l}\text { (5) } \\
\text { Resolution } \\
\text { (mas) }\end{array}$ & $\begin{array}{c}(6) \\
\text { rms noise } \\
\left(\mu \mathrm{Jy} \mathrm{beam}^{-1}\right)\end{array}$ \\
\hline \multirow[t]{2}{*}{1998 Mar 27} & 4.991 & VLBA,VLA & 62 & $3.19 \times 1.76, \mathrm{pa}-10^{\circ}$ & 102 \\
\hline & 8.421 & VLBA, VLA & 316 & $1.62 \times 1.22, \mathrm{pa}-1^{\circ}$ & 70 \\
\hline \multirow[t]{3}{*}{2002 May 16} & 4.991 & VLBA, $\mathrm{VLA}^{\mathrm{a}}, \mathrm{Eb}^{\mathrm{b}}$ & 30 & $3.24 \times 0.95, \mathrm{pa}-37^{\circ}$ & 48 \\
\hline & 8.421 & $\mathrm{VLBA}, \mathrm{VLA}^{\mathrm{a}}, \mathrm{Eb}^{\mathrm{b}}$ & 37 & $1.36 \times 0.48, \mathrm{pa}-18^{\circ}$ & 69 \\
\hline & 15.365 & $\mathrm{VLBA} \mathrm{VLA}^{\mathrm{a}}, \mathrm{Eb}^{\mathrm{b}}, \mathrm{GBT}^{\mathrm{c}}$ & 142 & $0.95 \times 0.46, \mathrm{pa}-19^{\circ}$ & 42 \\
\hline
\end{tabular}

a The VLA had a reduction of $\sim 15 \%$ in the effective time on source in order to phase the array and to observe a flux-density calibrator.

${ }^{\mathrm{b}} \mathrm{Eb}$ had a reduction of $\sim 50 \%$ in the effective time on source for pointing checks and because the galaxy set at Eb part-way through the observing session.

${ }^{\mathrm{c}}$ The GBT observed only at $15.3 \mathrm{GHz}$ in 2002 . 
Table 2. Radio Component Flux Densities ${ }^{\mathrm{a}}$

\begin{tabular}{lccccc}
\hline \hline & $(2)$ & $(3)$ & $(4)$ & $(5)$ & $(6)$ \\
Component & $\begin{array}{c}\Delta \alpha \\
(\mathrm{mas})\end{array}$ & $\begin{array}{c}\Delta \delta \\
(\mathrm{mas})\end{array}$ & $\begin{array}{c}S_{5} \\
(\mathrm{mJy})\end{array}$ & $\begin{array}{c}S_{8} \\
(\mathrm{mJy})\end{array}$ & $\begin{array}{c}S_{15} \\
(\mathrm{mJy})\end{array}$ \\
& & $\underline{1998}$ & & & \\
& $\ldots$ & $\ldots$ & $\ldots$ & $\ldots$ & $\ldots$ \\
D3a & 0.00 & 0.00 & $2.9 \pm 0.2$ & $2.7 \pm 0.2$ & $\ldots$ \\
D3b & $+1.16 \pm 0.20$ & $+0.02 \pm 0.20$ & $\ldots$ & $0.7 \pm 0.1$ & $\ldots$ \\
D3c & $+68.15 \pm 0.10$ & $-0.19 \pm 0.10$ & $\ldots$ & $1.7 \pm 0.1$ & $\ldots$ \\
E1 & & $\underline{2002}$ & & & \\
& $0.90 \pm 0.06$ & $0.01 \pm 0.12$ & $\ldots$ & $\ldots$ & $0.7 \pm 0.1$ \\
D3a & 0.00 & $4.0 \pm 0.3$ & $3.2 \pm 0.2$ & $3.0 \pm 0.4$ \\
D3b & $+1.51 \pm 0.06$ & $+0.37 \pm 0.10$ & $\ldots$ & $0.8 \pm 0.1$ & $0.5 \pm 0.1$ \\
D3c & $+68.33 \pm 0.06$ & $-0.52 \pm 0.09$ & $3.0 \pm 0.2$ & $1.5 \pm 0.1$ & $0.7 \pm 0.1$ \\
E1 & & & & & \\
\hline
\end{tabular}

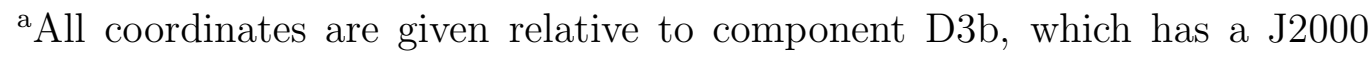
position (good to 1 mas) of $\alpha=12 \mathrm{~h} 10 \mathrm{~m} 32.5758 \mathrm{~s}, \delta=39^{\circ} 24^{\prime} 21.060^{\prime \prime}$. 